\title{
LTP Allergy: a pragmatic and reasonable approach in clinical practice
}

\author{
${ }^{1}$ Ambulatorio di Allergologia, Casa di Cura Villa Montallegro, Genova, Italy \\ ${ }^{2}$ Pediatric Allergy, Istituto Giannina Gaslini, Genoa, Italy
}

\section{KeYWORDS}

LTP; allergy; sensitization; adults;

children

\section{Corresponding author}

Ambulatorio di Allergologia,

Casa di Cura Villa Montallegro

Via P. Boselli 5

16146 Genova

Phone: +390523997390

E-mail: gio.cip@libero.it

\section{Doi}

10.23822/EurAnnACI.1764-1489.75

\section{To the Editor}

We read with great interest the article by Asero and colleagues concerning LTP allergy (1). We have to thank these authors for investigating a topic that is very intriguing and clinically relevant in our Country (2). The proposed pragmatic approach, based on encouraging LTP-sensitized patients to go on eating all foods containing LTP that they had tolerated until the first visit, is, in our opinion, very reasonable and represents a correct management of these patients. Consistently, we fully agree with the authors about the concept that going on eating well tolerated foods is a correct practice from an immunological point of view, particularly in children. Indeed, as suggested by Asero and colleagues, to continue eating the tolerated food constitutes a physiological sort of "natural, attenuated oral immunotherapy" (1). On the contrary, avoiding a tolerated food could promote true clinical allergy onset because of impaired immunological tolerance as a consequence of failed allergen exposure.

However, another crucial question is how to behave with subjects showing mere sensitization to LTP molecules. This eventuality happens more and more in allergy clinics, especially when using molecular allergy diagnostics. Actually, the most frequent mistake, made also by some allergist, is to confuse mere sensitization with true allergy. The consequence is the prescription of restriction diets that are useless or even dangerous as defined above. Another regrettable approach is to a priori recommend strict avoidance of all LTP-containing foods in the belief of the risk of potential anaphylaxis. One further common (mal)practice is the prescription of self-administered epinephrine even in the presence of a mere sensitization as a consequence of defensive medicine.

We are deeply convinced that allergy approach towards LTP-sensitized patients should be based on a rational and logical attitude based on immunological knowledge. In other words, LTP-sensitized subjects (such as without clinical reaction) could ingest all tolerated foods, at least until evident symptoms appears. A correct medical approach should aim at improving patient's "engagement" on his real clinical condition to distinguish between tolerance and symptoms. Information and education should always be part of the medical visit.

Another interesting issue is the protective role exerted by co-sensitization to some pan-allergenic molecules, i.e. PR-10 or 
profilins. In this regard, local geographic factors may have a relevant impact on the sensitization profile and the natural history of food allergy onset. Actually, the Genoa area model may be paradigmatic. Even though Genoa is placed in a birch-free geographic area, Betulaceae pollens sensitization (mainly to hazelnut tree and hornbeam [Ostrya carpinifolia]) is very common and its prevalence is increasing (3). Consequently, co-sensitization to Bet v 1 and LTP molecules is quite common (4). Notably, it has been recently reported that a group of adult patients with allergic rhinitis to Parietaria allergy (i.e. an LTP-molecule) did not report any severe adverse reactions to LTP-containing foods (5). Interestingly, $44 \%$ of these patients were co-sensitized to PR-10 allergen molecules and $16 \%$ to profilin ones. However, age may be another critical factor involved in the progression from sensitization to allergy. In this regard, we recently reported that severe LTP allergy may occur in children with allergic rhinitis due to Parietaria pollen and with Pru p 3 sensitization (manuscript submitted). On the other hand, a large quote of Pru p 3-sensitized children had no clinical allergy to LTP molecules. It is well known that the age has a relevant impact on $\mathrm{IgE}$ production, both concerning pollen and food allergens $(6,7)$. A final point should be considered: the level of serum allergen-specific IgE; it is usually considered a valuable biomarker for defining allergy diagnosis (8), symptom severity (9), and responsiveness prediction to allergen-specific immunotherapy (10). Unfortunately, as reported by the authors themselves and anecdotally, the serum level of IgE to LTP molecules does not predict the risk of the evolution toward clinical reaction to LTP foods (1).

In conclusion, we believe, in agreement with Asero and colleagues, that LTP sensitization and allergy should be correctly managed on an individual basis both in adult and paediatric patients to improve wellness and quality of life. Moreover, some variables should be carefully addressed, including age, area of residence, co-sensitization, co-morbidity, and sports practice after eating.

\section{References}

1. Asero R, Piantanida M, Pravettoni V. Allergy to LTP: to eat or not to eat sensitizing foods? A follow-up study. Eur Ann Allergy Clin Immunol 2018; 50:156-162.

2. Asero R, Antonicelli L, Arena A, et al. EpidemAAITO: features of food allergy in Italian adults attending allergy clinics: a multicentre study. Clin Exp Allergy 2009; 39:547-555.

3. Negrini AC, Negrini S, Giunta V, et al. Thirty year survey on airborne pollen concentrations in Genoa (Italy): relationship with sensitizations, meteorological data, and air pollution. Am J Rhinol Allergy 2011; 25:232-241.

4. Ciprandi G, Comite P, Mussap M, et al. Profiles of birch sensitization (Bet v 1, Bet v 2, Bet v 4) and oral allergy syndrome across Italy. J Inv All Clin Immun 2016; 26:244-248.

5. Ciprandi G, Ferrero P, Comite P. The pragmatic relevance of Pru p 3 sensitization in patients with pollen allergy. Rev Francaise Allergologie 2018 (in press).

6. Tosca MA, Silvestri M, Olcese R, et al. The impact of age on serum allergen-specific IgE to inhaled molecular components. Allergol Immunopathol 2017; 45265-45271.

7. Tosca MA, Silvestri M, Olcese R, et al. Allergen-specific IgE to food molecular components and age: from early childhood to adulthood. Allergol Immunopathol 2017; 45:87-92.

8. Alesina R, DeAmici M, Ciprandi G. Serum IgE discriminates allergy from sensitization better than skin testing. Allergol Immunopathol 2014; 42:171-173.

9. Corsico AG, DeAmici M, Ronzoni V, et al. Allergen-specific IgE and allergic rhinitis severity. Allergy and Rhinology 2017; 8:1-4.

10. Ciprandi G. Serum IgE as biomarker for predicting allergen immunotherapy effectiveness. J Allergy Clin Immunol 2017; 139:2029. 